\title{
Neonatal outcome after prolonged rupture of the membranes starting in the second trimester
}

\author{
M BLOTT AND A GREENOUGH \\ Department of Child Health, King's College Hospital, London
}

SUMMARY The neonatal outcomes of 30 pregnancies that were complicated by premature and prolonged rupture of the membranes that had started in the second trimester of pregnancy, were reviewed. The neonatal mortality was $11(36 \%)$, the main cause of death being pulmonary hypoplasia. Two infants died of sepsis, but these were the only proved episodes of maternal or fetal infection. Of the survivors $27 \%$ developed compressive limb abnormalities, all of which responded to passive physiotherapy. Pulmonary hypoplasia was significantly associated with earlier onset of rupture of the membranes, and the absence of fetal breathing movements. Compressive limb abnormalities were significantly associated with longer periods of oligohydramnios. We conclude that premature rupture of the membranes, even with onset in the second trimester, may be associated with a favourable outcome and this may be predicted by the persistence of fetal breathing movements. We therefore, recommend expectant management of such pregnancies, but suggest elective delivery at 34 weeks to limit fetal exposure to uterine compression and minimise the risks of prematurity.

Premature rupture of the membranes is a common complication of pregnancy. ${ }^{1}$ When it occurs in the second trimester the prognosis is poor, ${ }^{2}$ and as a consequence termination of the pregnancy is often recommended. The neonates may have pulmonary hypoplasia, altered facies, and aberrant limb development (Potter's syndrome). ${ }^{3}$ Premature rupture of the membranes permits chronic drainage of amniotic fluid resulting in oligohydramnios, so such abnormalities may be the result of prolonged compression of the fetus by the uterus in the absence of the usual 'cushion' of amniotic fluid. ${ }^{4}$ Pulmonary hypoplasia has been reported after oligohydramnios of only six days' duration, ${ }^{5}$ but it has been suggested that compressive abnormalities are more likely to develop after chronic reduction in the amount of amniotic fluid. ${ }^{2}$ Our preliminary report, however, included normal survivors of pregnancies complicated by severe oligohydramnios that had existed for as long as 17 weeks. $^{6}$

The aim of the present study was to follow prospectively a larger series of pregnancies complicated by prolonged rupture of the membranes that had started in the second trimester in an attempt to find out the neonatal mortality and morbidity. We hoped to determine the association between the onset and duration of rupture of the membranes and mortality and morbidity, and find out if the persistence of fetal breathing movements is a reliable indicator of favourable neonatal outcome. ${ }^{6}$

\section{Patients and methods}

During a period of one year all pregnancies with rupture of the membranes of longer than two weeks' duration occurring in the second trimester were eligible for entry into the study. The various options in management and the prognosis of pregnancies complicated by premature rupture of the membranes were carefully explained to the patients referred to our unit and their partners. If a patient then decided to be treated expectantly she was entered into the study.

The patient was admitted to the ward, where she was carefully monitored for signs of developing infection. Spontaneous premature labour was not inhibited. If the patient developed signs of chorioamnionitis, immediate delivery was undertaken ${ }^{7}$; otherwise, elective delivery was carried out at 32-34 weeks as a compromise between limiting the duration of uterine compression on the fetus, and not exposing the neonate to the risks of extreme prematurity. During admission ultrasound examinations were performed at weekly intervals (and more 
often if indicated) to assess fetal growth, the volume of amniotic fluid, and fetal breathing movements. Oligohydramnios was defined as the absence of a pool of amniotic fluid greater than one centimetre in diameter in a vertical plane. ${ }^{8}$ Fetal breathing was said to be present if a continuous period of chest wall movement persisted for longer than 60 seconds in any 30 minute period, with a breath to breath interval of less than six seconds. ${ }^{9}{ }^{10}$ All ultrasound examinations were carried out within two hours of the patient receiving a concentrated dose of glucose.

After delivery the infants were carefully examined. The presence of physical deformities associated with the oligohydramnios tetrad were noted. ${ }^{11}$ Pulmonary hypoplasia was diagnosed if the infant required high peak inspiratory pressures (in excess of $30 \mathrm{~cm} \mathrm{H}_{2} \mathrm{O}$ ) during positive pressure ventilation both at resuscitation and subsequently, and if respiratory support was necessary for longer than one month. ${ }^{6}$ Additional evidence was given by a chest radiograph (reported without clinical details) that was compatible with lungs of small volume. Necropsy was requested in the event of fetal or neonatal death. Pulmonary hypoplasia was confirmed if the lung:body weight ratio was less than 0:012 and the radial alveolar count less than or equal to $4 \cdot 1 .^{12}$

The infants were divided into three groups for analysis: group 1 comprised neonates with clinical or pathological evidence of pulmonary hypoplasia or both, group 2 comprised neonates with compressive limb abnormalities but no evidence of pulmonary hypoplasia, and group 3 comprised neonates with neither pulmonary hypoplasia nor limb abnormalities.

The significance of differences between the groups in the duration of rupture of the membranes and gestational age at its onset and at delivery were calculated by the Wilcoxon rank sum test.

Permission for this study was granted by the King's College Hospital ethics committee.

\section{Results}

During the year 30 patients were eligible for entry into the study. All chose to be managed expectantly and were therefore included. The pregnancies were all singletons without other complications. The mean gestational age at rupture of the membranes was $21 \cdot 3$ weeks (range 15-28).

Infection occurred in only two cases; both neonates were from group 3 . In the first case although amniocentesis had shown white cells but no organisms, the infant died from infection in the first 48 hours of life after delivery by emergency caesarean section. The mother made a good recovery and had no evidence of postnatal infection. In the second case delivery by emergency caesarean section was undertaken at 28 weeks' gestation for placenta praevia. Although infection was not suspected antenatally the infant died early in the neonatal period from overwhelming sepsis. There were no other cases of maternal or neonatal infection.

Most of the patients delivered after the onset of spontaneous labour, 10 by the vertex and eight by assisted breech delivery. Six patients were delivered by emergency caesarean section; in four cases this was for fetal distress after the onset of spontaneous labour. One infant was born vaginally after induction of labour, and the remaining five babies were delivered by elective caesarean section between 32 and 34 weeks' gestation.

Eleven neonates died, an overall mortality of $36 \%$, and in eight cases death was attributable to pulmonary hypoplasia. Of the 30 infants, $14(46 \%)$ had physical deformities associated with oligo-

Table Mean (range) gestational ages at onset of rupture of membranes and at delivery, and duration of rupture

\begin{tabular}{llll}
\hline & $\begin{array}{l}\text { Onset } \\
\text { (weeks) }\end{array}$ & $\begin{array}{l}\text { Delivery } \\
\text { (weeks) }\end{array}$ & $\begin{array}{l}\text { Duration } \\
\text { (weeks) }\end{array}$ \\
\hline Group 1 & $17(15-22)$ & $27(24-34)$ & $10(4-19)$ \\
Group 2 & $18(17-23)$ & $31(28-35)$ & $13(7-15)$ \\
Group 3 & $24(18-28)$ & $29(25-35)$ & $5(2-10)$ \\
\hline
\end{tabular}

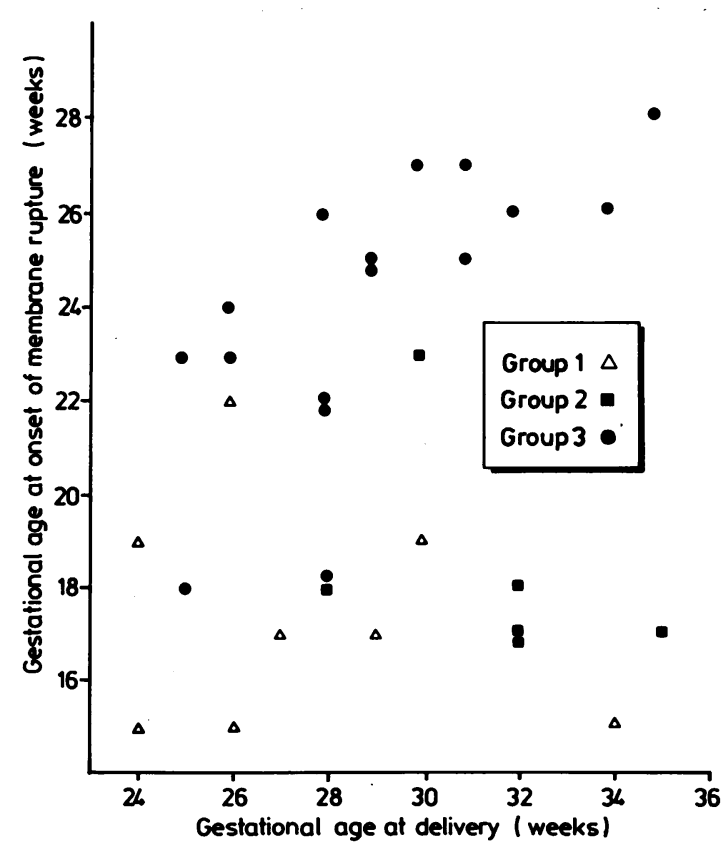

Figure Gestational ages at onset of rupture of the membranes and delivery in the three groups. 
hydramnios $^{11}$ but no survivor had limb abnormalities that required surgical correction.

The mean gestational ages at rupture of the membranes and delivery in the three groups are shown in the table and the figure. The gestational age of the onset of rupture of the membranes was significantly earlier in groups 1 and 2 than in group 3 $(p<0 \cdot 01)$. The duration of rupture of the membranes was only significantly longer when group 2 was compared with group $3(\mathrm{p}<0 \cdot 01)$. Infants in group 2 were delivered at a later gestational age than those in group $1(\mathrm{p}<0.05)$, but there were no other significant differences in the timing of delivery among the three groups. All 30 pregnancies had similar degrees of oligohydramnios, but only in group 1 were fetal breathing movements never seen on ultrasound examination $(p<0 \cdot 01)$.

\section{GROUP 1}

Eight infants had clinical evidence of pulmonary hypoplasia. Despite high peak inspiratory pressures (mean 38, range $30-55 \mathrm{~cm} \mathrm{H}_{2} \mathrm{O}$ ), satisfactory oxygenation was never achieved, and all eight infants died within 48 hours of birth. Confirmation of pulmonary hypoplasia was obtained at necropsy in seven cases. The request for necropsy was refused by the parents of the other child. This infant required a maximum peak inspiratory pressure of 55 $\mathrm{cm} \mathrm{H}_{2} \mathrm{O}$ during artificial ventilation, developed bilateral pneumothoraces, and died at 24 hours of age; satisfactory oxygenation was never achieved. All eight infants had Potter's facies and fixed flexion deformities.

\section{GROUP 2}

Six infants were born with compressive limb abnormalities without clinical evidence of pulmonary hypoplasia. Three of them did develop the respiratory distress syndrome and required ventilatory. support, but none required peak inspiratory pressures in excess of $30 \mathrm{~cm} \mathrm{H}_{2} \mathrm{O}$. The remaining three infants required no respiratory support in the neonatal period. The six infants had bilateral talipes, and three had mild Potter's facies, but none had congenital dislocation of the hips. All limb abnormalities responded to physiotherapy, and none required surgical correction.

\section{GROUP 3}

Sixteen infants had no abnormalities attributable to oligohydramnios. Although 11 infants required artificial ventilation for respiratory distress syndrome, the maximum peak inspiratory pressure used was less than $30 \mathrm{~cm} \mathrm{H}_{2} \mathrm{O}$ (mean 18, range $12-25 \mathrm{~cm} \mathrm{H}_{2} \mathrm{O}$ ). Only one infant required prolonged ventilation. She was born at 25 weeks' gestation and developed severe respiratory distress syndrome, and a patent ductus arteriosus that required surgical ligation. Three of the 16 infants died, all three having been delivered before 34 weeks' gestation. At necropsy there was no evidence of pulmonary hypoplasia. One had delivered after the spontaneous onset of labour at 26 weeks' gestation and died of respiratory distress syndrome. The other two infants died of sepsis.

\section{Discussion}

Fatal pulmonary hypoplasia following premature rupture of the membranes is common, occurring in $26 \%$ of cases in this series and in $33 \%^{5}$ and $21 \%^{2}$ in two other studies. The gestational age at the time of rupture of the membranes was significantly associated with the development of pulmonary hypoplasia, and confirmed earlier findings that pulmonary hypoplasia was more common when rupture of the membranes occurred before 26 weeks' gestation. ${ }^{213}$ Critical lung development occurs before 26 weeks' gestation, ${ }^{14}$ and so damage after this time would be expected to have less effect on pulmonary growth and function. In $23 \%$ of neonates born without pulmonary hypoplasia, however, rupture of the membranes had occurred before 20 weeks' gestation.

There are several possible explanations for the association between pulmonary hypoplasia and oligohydramnios. Intrathoracic compression of the developing fetal lung, as associated with congenital diaphragmatic hernia and pleural effusions, often results in pulmonary hypoplasia. ${ }^{15} 16$ It may therefore be that extra thoracic compression of the fetal chest by the uterine wall in the absence of the usual amniotic fluid cushion could have prevented normal lung growth. In this series, however, all pregnancies had the same degree of oligohydramnios (assessed by ultrasound examination) but only a proportion of the fetuses developed pulmonary hypoplasia. Ultrasound assessment of the volume of amniotic fluid is comparatively inaccurate, and it may be that minor differences in the volume of amniotic fluid that were undetectable by ultrasonography were responsible for the variation in outcome.

Fetal breathing movements have been shown to be critical for normal lung growth. ${ }^{17}$ In this series the absence of sustained respiratory activity was associated with the development of pulmonary hypoplasia. Though this seems contrary to findings reported recently, ${ }^{18}{ }^{19}$ this discrepancy is explained by the different definitions of fetal breathing movements used in the three studies. In the present series we considered fetuses to be breathing only if an episode of chest wall movement was continuous for at least 60 seconds with no breath to breath interval 
of greater than six seconds; this definition has been used extensively. ${ }^{8} 91020$ The other two studies ${ }^{18} 19$ accepted fetuses to be breathing if respiratory activity persisted for only six seconds. We felt that that definition was unacceptable, because short bursts of fetal breathing movements or gasping can occur in severely hypoxic infants, ${ }^{21} 22$ and thus cannot be used as an indicator of fetal well being. Indeed, in our series, such 'gasping' respiration was witnessed in fetuses who subsequently died of pulmonary hypoplasia.

Though the neonates that died of pulmonary hypoplasia had undergone earlier rupture of the membranes, other neonates with similar early onsets of rupture of the membranes had apparently normal lung development. Thus the gestational age at onset of rupture cannot be the sole explanation for the development of pulmonary hypoplasia. The length of time after the membranes had ruptured did not appear to be critical for the development of pulmonary hypoplasia as this was longer among the infants who had normal lung growth (group 2). Neither can the timing of delivery be incriminated, because there was no significant difference in the gestational age at delivery between infants in groups 1 and 3 . Our results suggest, therefore, that the development of pulmonary hypoplasia in pregnancies with early rupture of the membranes is associated with the disappearance of fetal breathing movements. Sustained periods of fetal breathing seem to preserve lung growth even when rupture occurs within the 'critical' period of lung growth.

Pulmonary hypoplasia after oligohydramnios can occur in isolation without limb abnormalities. ${ }^{23}{ }^{24}$ In this series, however, there were infants with flexion deformities of the limbs with normal lung growth, which is unusual. ${ }^{425}$ The limb abnormalities were significantly associated with both the time of onset and the duration of oligohydramnios. Fetal immobilisation in utero as a consequence of paralysing drugs given to the mother, or musculoskeletal disorders of the fetus, result in fixed flexion deformities. ${ }^{26}$ It is likely that the association between oligohydramnios and fixed flexion deformities is caused by prolonged compression by the uterus resulting in fetal immobilisation. This hypothesis is supported by the finding of a significantly longer duration of oligohydramnios and later gestation at delivery in this group of infants. In our series none of the surviving infants required more than passive physiotherapy to correct the limb abnormalities. In all cases delivery occurred before 35 weeks' gestation, and it may be that this prevented progression of the limb abnormalities.

Infection has been proposed as a cause of premature rupture of the membranes ${ }^{27}$ and as a consequence there has been concern that expectant management of such pregnancies might lead to serious maternal complications. ${ }^{1}$ In this series we excluded all pregnancies with rupture of the membranes of less than two weeks' duration. This may have prevented us including pregnancies for expectant management who had rupture as a result of intrauterine infection. This series has shown that long standing rupture of the membranes is not often associated with infection. We had only one case where infection was suspected antenatally, and this was not confirmed in the mother though the neonate died of sepsis. One other infant died early in the neonatal period of sepsis; the mother never exhibited signs of infection either in the antenatal or postnatal period.

Premature rupture of the membranes before 20 weeks' gestation poses a difficult problem in management. A large proportion of such pregnancies will either abort spontaneously, or develop infection shortly after rupture of the membranes necessitating termination of the pregnancy. In the present study we included only pregnancies in which rupture of the membranes had lasted for at least two weeks' gestation; thus epidemiological statements about the outcome of all pregnancies with rupture of the membranes before 20 weeks' gestation cannot be made. It is interesting to note, however, that among our patients with rupture of the membranes before 20 weeks' gestation, none developed sepsis and though $50 \%$ died from pulmonary hypoplasia the others survived intact or with minor orthopaedic problems.

The results of this study show that premature and prolonged rupture of the membranes can be associated with intact neonatal survival, although the mortality of $26 \%$ and morbidity of $27 \%$ is considerable. The presence of fetal breathing movements, even in pregnancies with rupture of the membranes at as early as 18 weeeks' gestation was associated with normal lung growth and favourable outcome in all cases. Our results suggest that in such pregnancies expectant management is a reasonable course, but that delivery should be instituted electively before full term to reduce the possible morbidity caused by limb abnormalities.

Dr M Blott, research fellow, is supported by Action Research for the Crippled Child.

\footnotetext{
References

1 Gunn GC, Mishell DR, Morton DG. Premature rupture of the fetal membranes. A review. Am J Obstet Gynecol 1970;106: 469-73.

${ }^{2}$ Nimrod C, Varela-Gittings F, Machin G, Campbell D, Wesen-
} 
berg $\mathrm{R}$. The effect of very prolonged membrane rupture on fetal development. Am J Obstet Gynecol 1984;148:540-3.

${ }^{3}$ Perlman M, Williams J, Hirsch M. Neonatal pulmonary hypoplasia after prolonged leakage of amniotic fluid. Arch Dis Child 1976;51:349-53.

4 Thomas IT, Smith DW. Oligohydramnios, cause of the nonrenal features of Potter's Syndrome, including pulmonary hypoplasia. J Pediatr 1974;84:811-4.

5 Thibeault DW, Beatty EC, Hall RT, Bower SK, O'Neill DH. Neonatal pulmonary hypoplasia with premature rupture of fetal membranes and oligohydramnios. J Pediatr 1985;107:273-7.

6 Blott M, Greenough A, Nicolaides KH, Moscoso G, Gibb D, Campbell S. Fetal breathing movement as a predictor of favourable pregnancy outcome after oligohydramnios due to membrane rupture in the second trimester. Lancet 1987;ii: 129-31.

7 Daikoku NH, Kaltreider F, Khouzami VA, Spence M, Johnson JWC. Premature rupture of membranes and spontaneous preterm labour. Maternal endometritis risks. Obstet Gynecol 1982;59:13-20.

${ }^{\star}$ Manning FA, Platt LD, Sipos L. Antepartum fetal evaluation: Development of a fetal biophysical profile. Am J Obstet Gynecol 1980;136:787-95.

9 Vintzileos AM, Campbell WA, Ingardia CJ, Nochimson DJ. The fetal biophysical profile and its predictive value. Obstet Gynecol 1983;62:271-8.

10 Roberts AD, Griffin D, Mooney R, Cooper DJ. Campbell S. Fetal activity in 100 normal third trimester pregnancies. $\mathrm{Br} J$ Obstet Gynaecol 1980;87:480-4.

"Fantel AG, Shephard TM. Potter's Syndrome. Non-renal features induced by oligohydramnios. Am J Dis Child 1975;129: 1346-7.

12 Askenazi SS, Perlman M. Pulmonary hypoplasia: lung weight and radial alveolar count as criteria of diagnosis. Arch Dis Child 1979;54:614-8.

13 Moessinger AC, Collins MH, Baau WA, Rey HR, James LS. Oligohydramnios induced lung hypoplasia: the influence of timing and duration in gestation. Pediatr Res 1986;20:951-4.

14 Reid L. Lung growth in health and disease. Br J Dis Chest 1984; 78: $113-35$.

15 Adzick NS, Harrison MR, Glock PL, Nakayama DK, Manning FA, de Lorimier AA. Diaphragmatic hernia in the fetus.
Prenatal diagnosis and outcome in 94 cases. J Pediatr Surg 1985;20:357-61.

16 Murayama K, Jimbo T, Matsumoto Y, Mitsuishi C, Nishida H. Fetal pulmonary hypoplasias with hydrothorax. Am J Obstet Gynecol 1987;157:119-20.

17 Wigglesworth JS, Desai R. Is fetal respiratory function a major determinant of perinatal survival. Lancet 1982;i:264-7.

${ }^{18}$ Kilbride HW, Thibeault DW, Yeast J, Mavlik D, Grundy HO. Fetal breathing is not a predictor of pulmonary hypoplasia in pregnancies complicated by oligohydramnios. Lancet 1988;i: 305-6.

${ }^{19}$ Moessinger AC, Higgins A, Fox HE, Rey HR. Fetal breathing movements are not a reliable predictor of continued lung development in pregnancies complicated by oligohydramnios. Lancet 1987;ii:1297-9.

20) Manning FA, Platt $C D$. Fetal breathing and the abnormal contraction stress test. Am J Obstet Gynecol 1979;133:590-3.

21 Trudinger BJ, Lewis PJ, Pettit B. Fetal breathing patterns in intrauterine growth retardation. Br J Obstet Gynaecol 1979;86: 432-6.

${ }^{22}$ Patrick JE, Dalton KJ, Dawes GJ. Breathing patterns before death in fetal limbs. Am J Obstet Gynecol 1976;125:73-8.

${ }^{23}$ Kilbride HW, Thibeault DW, Yeast J, Mavlik D, Meade V, Grundy HO. Fatal pulmonary hypoplasia within 17 days of severe oligohydramnios at 28 weeks' gestation. Pediatr Res 1987;21:456A.

24 Thibeault DW, Beatty EC, Hall RT, Bower SK. Prolonged rupture of fetal membranes (PROM) and hypoplastic lungs without the oligohydramnios tetrad. Pediatr Res 1983;17:392A.

${ }^{25}$ Bain AD, Smith II, Gould IK. Newborn after prolonged leakage of liquor. Br Med J 1964;ii:593-8.

26 Vago RH. Arthrogryposis following treatment of maternal tetanus with muscle relaxants. Arch Dis Child 1970;45:477-9.

27 Schreiber J, Benedette T. Conservative management of preterm premature rupture of the fetal membranes in a low socioeconomic population. Am J Obstet Gynecol 1980;136:92-6.

Correspondence to Dr Anne Greenough, Department of Child Health, King's College Hospital, Denmark Hill, London SE5 9RX.

Accepted 27 June 1988 